Scientific Spokesman:

A. K. Mann

Department of Physics University of Pennsylvania Philadelphia, Pa. 19104

FTS/Off-net : $\quad 215-597-3311$ 594-8141

\title{
SEARCH FOR A NEW CLASS OF PENETRATING MASSIVE PARTICLES AT C-O
}

\section{Submitted by}

J. Pilcher and A. Zylberstejn University of Chicago

D. Cheng, C. Rubbia and L. Sulak Harvard University

\section{W. Ford, T.X. Ling and A.K. Mann} University of Pennsylvania

A. Benvenuti, D. Cline, R. Imlay and D.D. Reeder University of Wisconsin 
We wish to search for a new class of penetrating, massive particles that might be directly produced in high energy proton collisions with the foil target at $\mathrm{C}_{0}$. The search is based on the following ideas.

1. Recent ISR data indicate that heavier hadronic particles are more copiously produced than light ones at large $p_{\perp}$. We speculate that the production of new heavy particles might also be relatively more probable at large $p_{\perp}$.

2. In any event it is feasible experimentally to search for the production of massive, non-strongly interacting particles with, say, $p_{\perp} \sim 4 \mathrm{GeV} / \mathrm{c}$, $p_{\text {tot }} \sim 40 \mathrm{GeV} / \mathrm{c}$, at an angle of 80-100 mrad. in the laboratory with the experimental arrangement shown in Fig. 1 . Note that the horizontal scale in Fig. 1 is 10 times larger than the vertical scale.

The scattered hadrons are absorbed in the primary shield as indicated. The Cherenkov counter, used as an anticounter, sets a lower limit on the mass of an unvetoed particle of $M \geq 1.3 \mathrm{GeV}$ $(\gamma<30)$. The solid iron bending magnets in conjunction with small drift chambers determine the momentum of the particles and also help to discriminate against low energy muons from $\pi$ and $k$ decays. A six-fold scintillation counter coincidence is made in addition to the Cherenkov counter veto.

3. It is particularly valuable to use the monotonically increasing energy of the circulating protons to search for threshold effects in the yield of particles that are recorded in the detector. This comes about naturally (following from experience in experiment E120) and, most important, provides a precision scan in energy every machine pulse. This is one of the most favorable features of particle searches at $\mathrm{C}_{0}$. 


\section{Rates and Backgrounds}

To show the sensitivity of this experiment we assume a cross section of $d \sigma / d \Omega_{1 \mathrm{ab}} \sim 10^{-30} \mathrm{~cm}^{2} /$ sterad for the production of new particles corresponding to $\mathrm{d} \sigma / \mathrm{d} \Omega \mathrm{c} . \mathrm{m} . \sim 10^{-32} \mathrm{~cm}^{2} / \mathrm{strad}$ for the new particle production suggested by the CERN-Rutherford-ISR experiment. The scintillation counters have an acceptance of $2.5 \times 10^{-5}$ sterad and the magnetic spectrometer has an acceptance of $\Delta \mathrm{p} / \mathrm{p} \simeq 0.2$. With $10^{8}$ interacting protons per pulse we expect 0.012 events/pulse. The observation of these events coupled with a threshold effect would be evidence for a new particle.

The corresponding background of $\pi$ 's produced with $p_{\perp}>4 \mathrm{GeV} / \mathrm{C}$ has a cross section of $d \sigma / d \Omega_{1 a b^{~}} 0^{-31}$ (at $E_{p}=200$ ) (taken from the $\pi^{\circ}$ data of Cool et al from the ISR). The decay probability of these $\pi^{\prime} \mathrm{s}$ is $10^{-3}$ for the expected decay length. Thus the rate for this background is $\sim 10^{-5} /$ pulse. This is probably too low a 1 imit on the background rates. A possibly more serious background will come from pions produced at smaller angles which multiply and interact in the absorber and then decay. We would however not expect this background to be more than 100 times the direct pion background.

The background rate from accidentals is somewhat more difficult to estimate but experience on running $\mathrm{E} 120$ at $\mathrm{C}_{0}$ indicates that this should also be manageable if careful precautions are taken in the electronics and scintillation counter design. It will be necessary to make target in-target out subtractions as well, using the R.F. structure of the machine to further reduce background. Again we have had good success in this regard in E120. 
In summary it seems possible to detect new massive semistrongly interacting particles, providing they are produced with a cross section of greater than $10^{-33} \mathrm{~cm}^{2} / \mathrm{strad}$. in the C.M. Evidence for the existence of a new particle would come from the observation of a threshold effect in the signal as a function of incident proton energy which would help also to discriminate against bizarre backgrounds.

Schedule

Within a few weeks after approval of this experiment, the experimental apparatus can be mounted in the tunnel at $\mathrm{C}_{0}$. It is expected that much of the existing electronics equipment for El 20 would be useful in this experiment. 


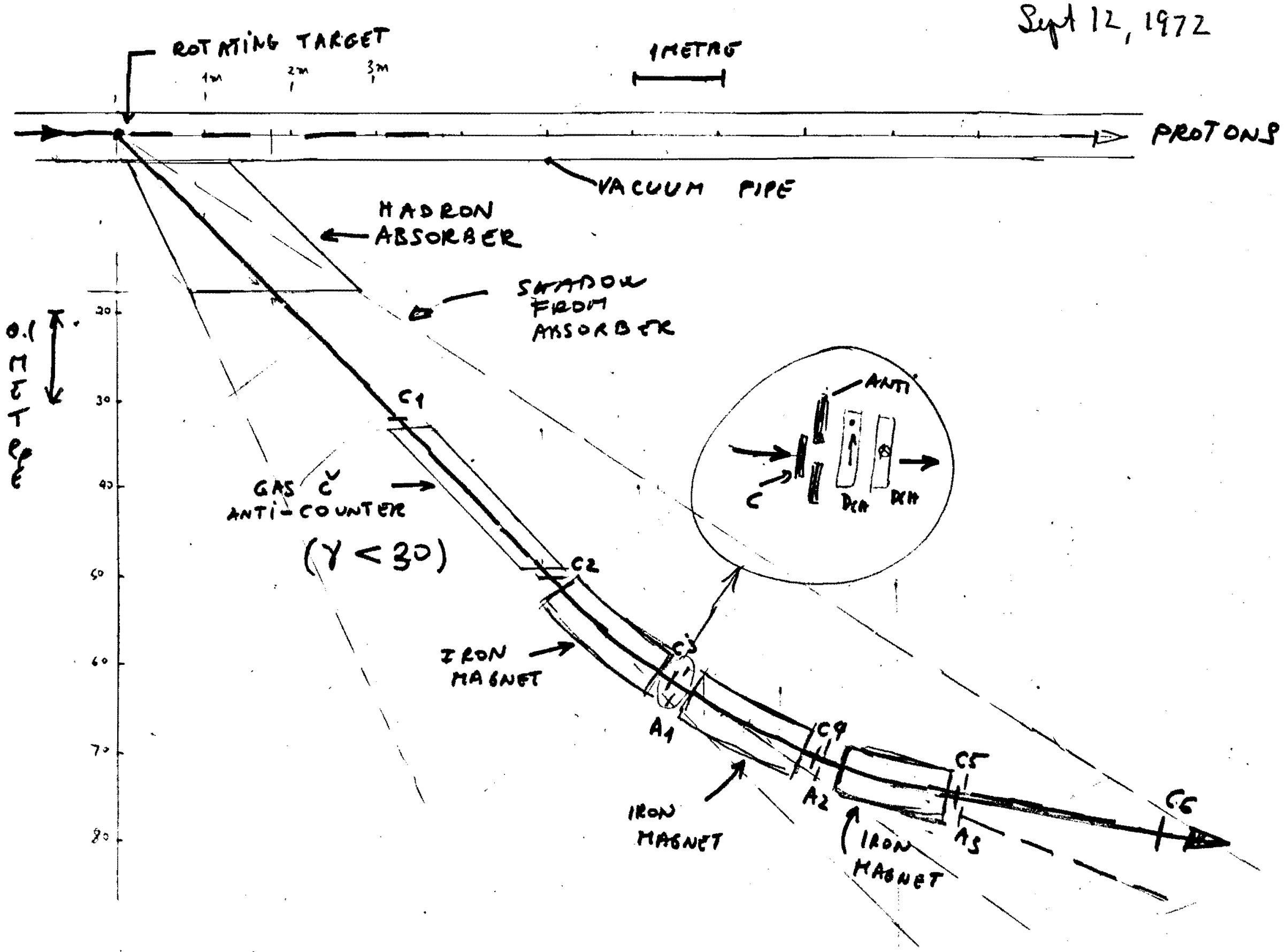




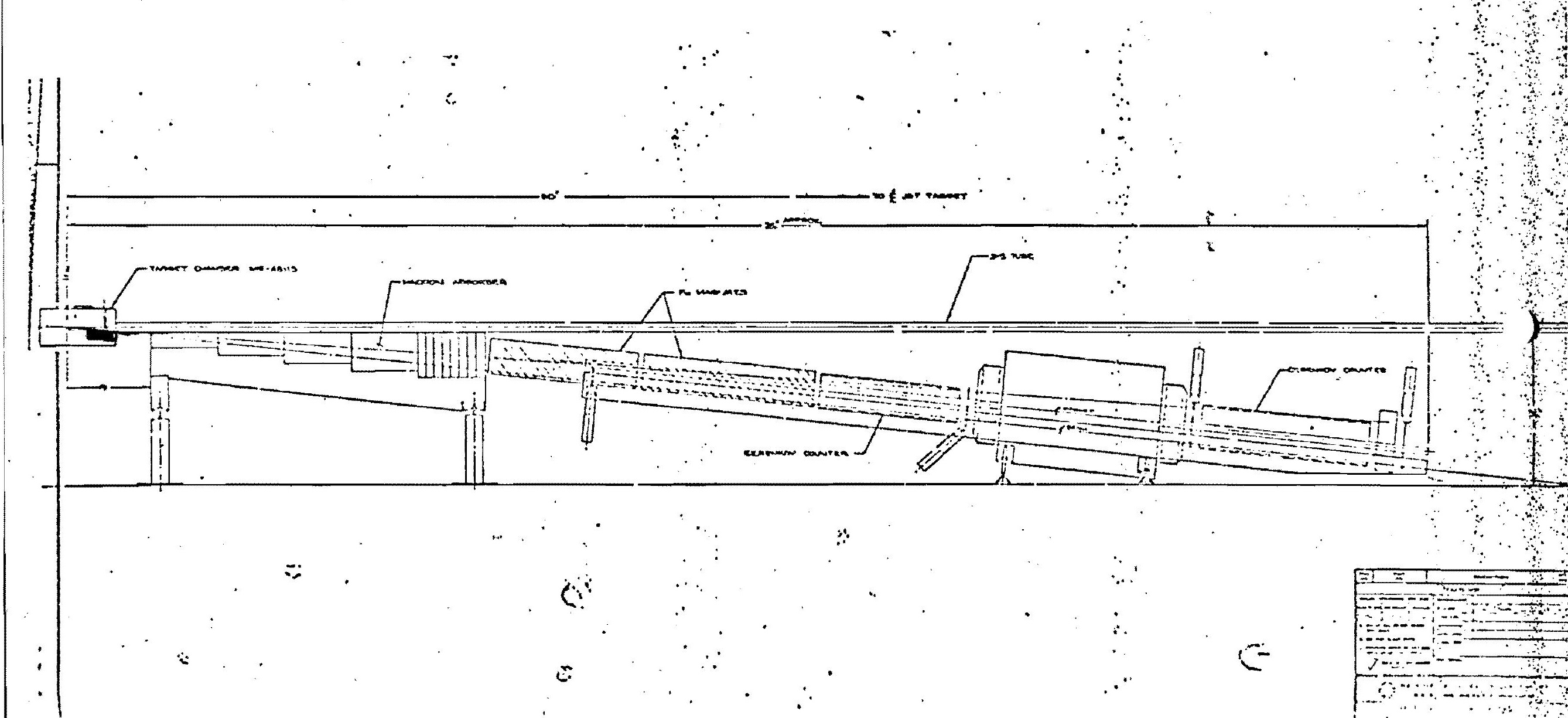




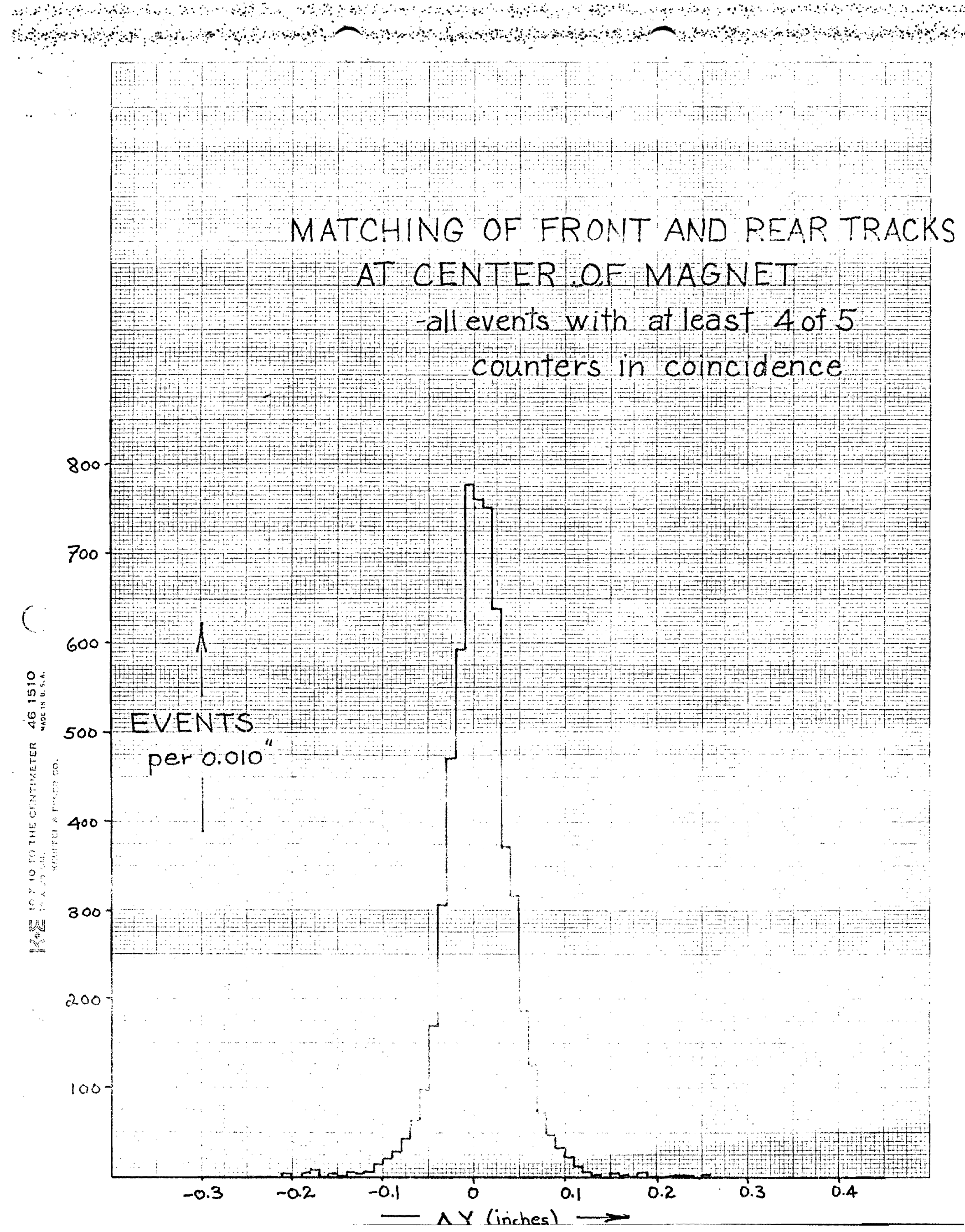


$4 \mathrm{~m}$ as

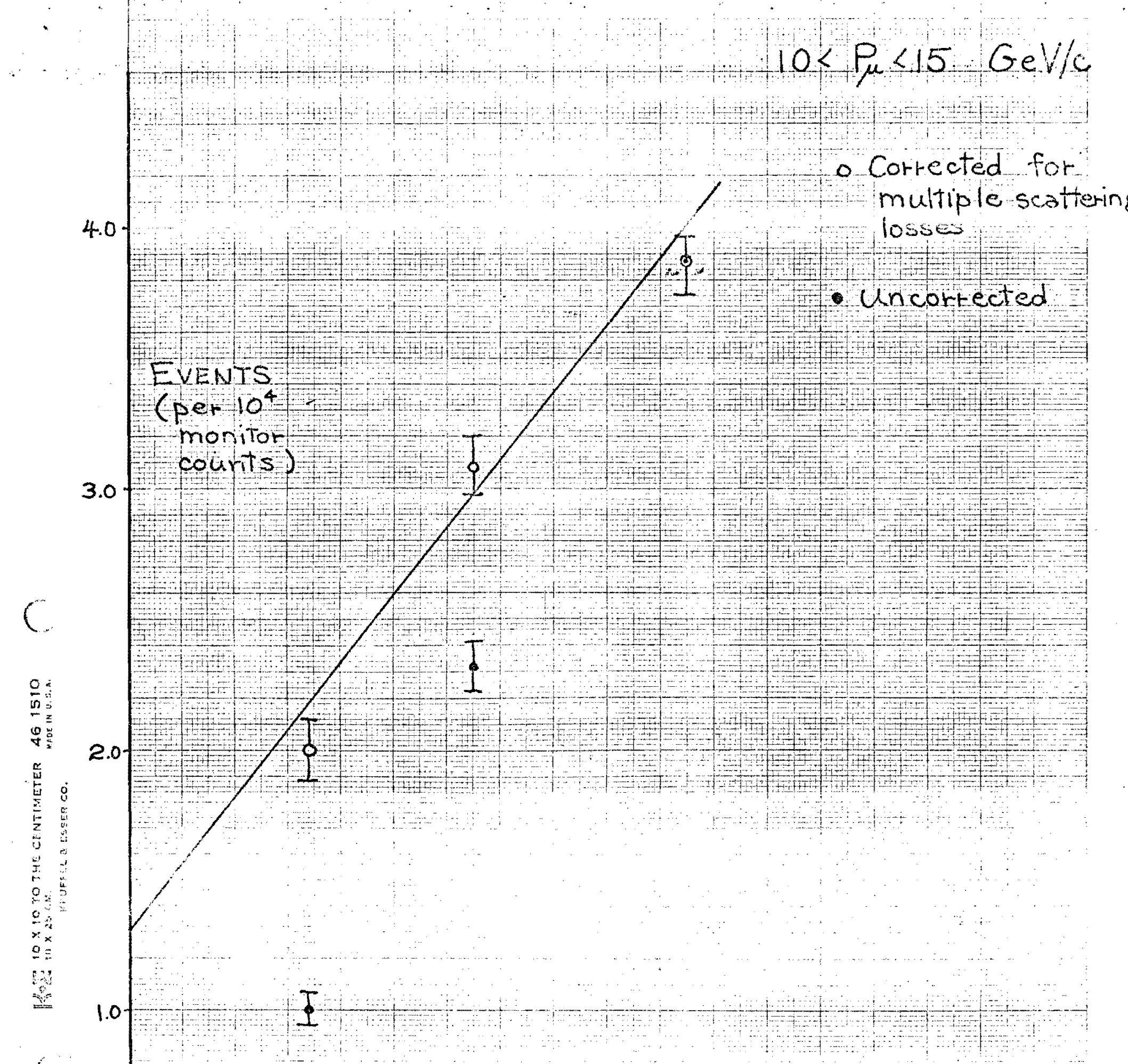




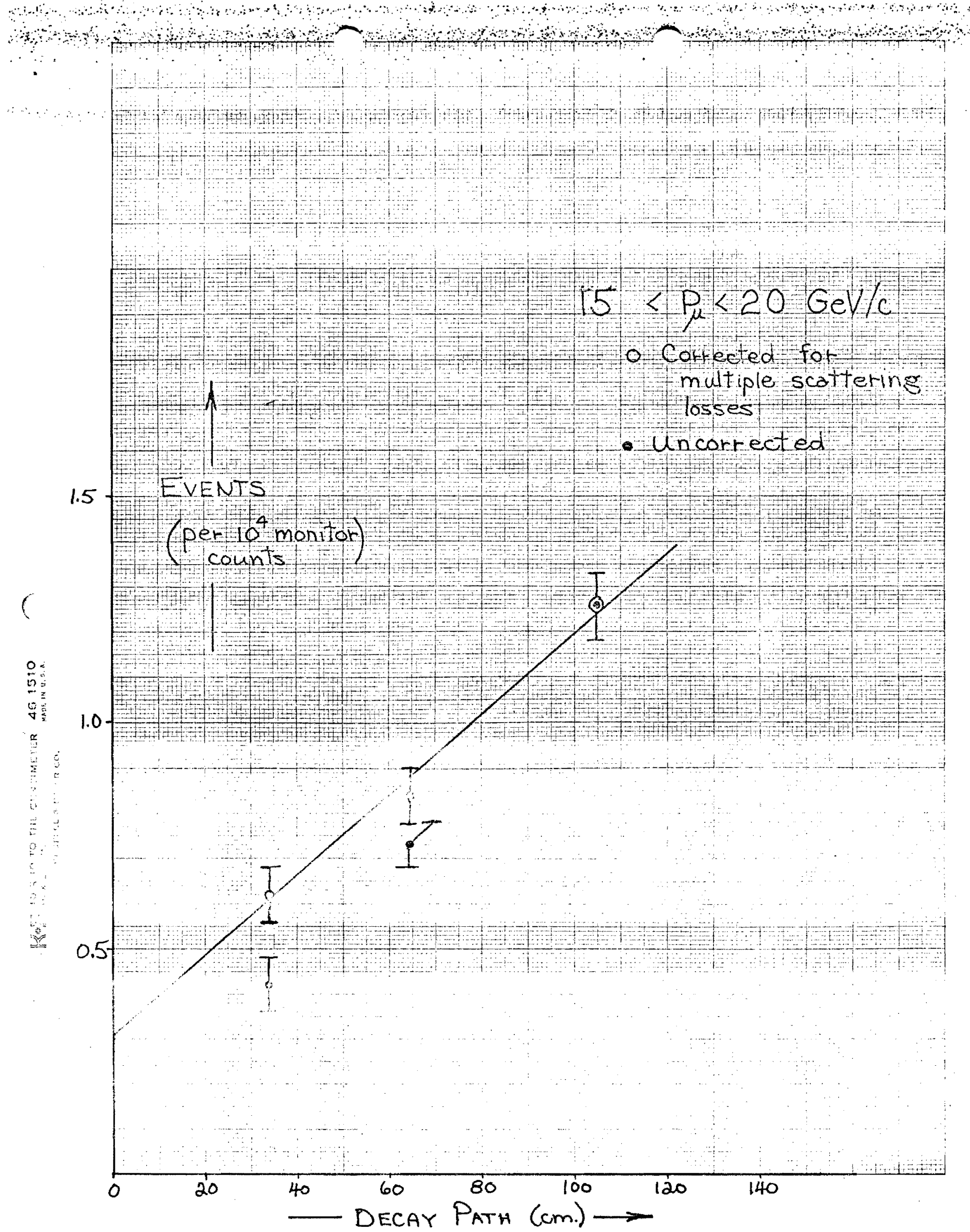




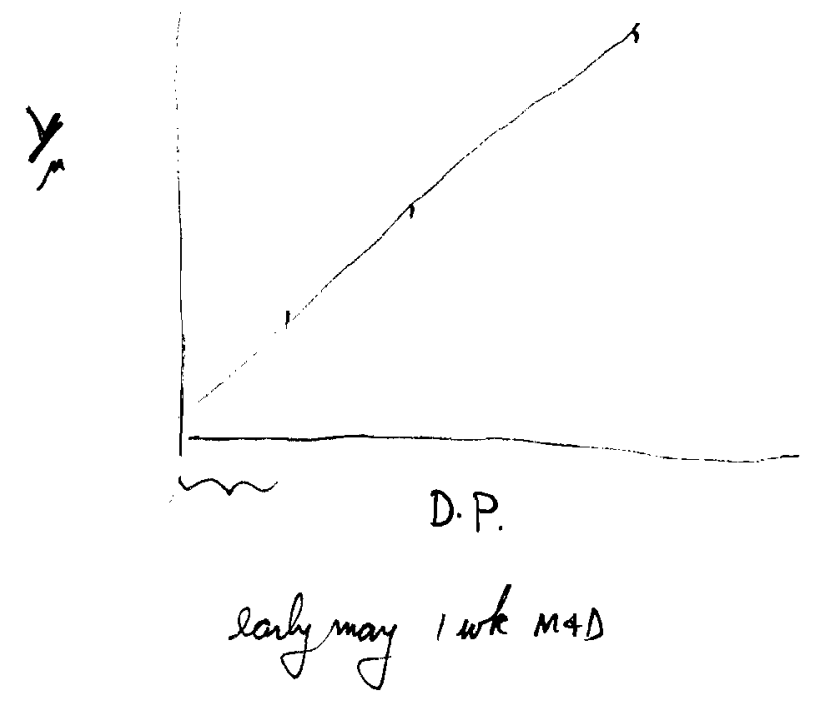



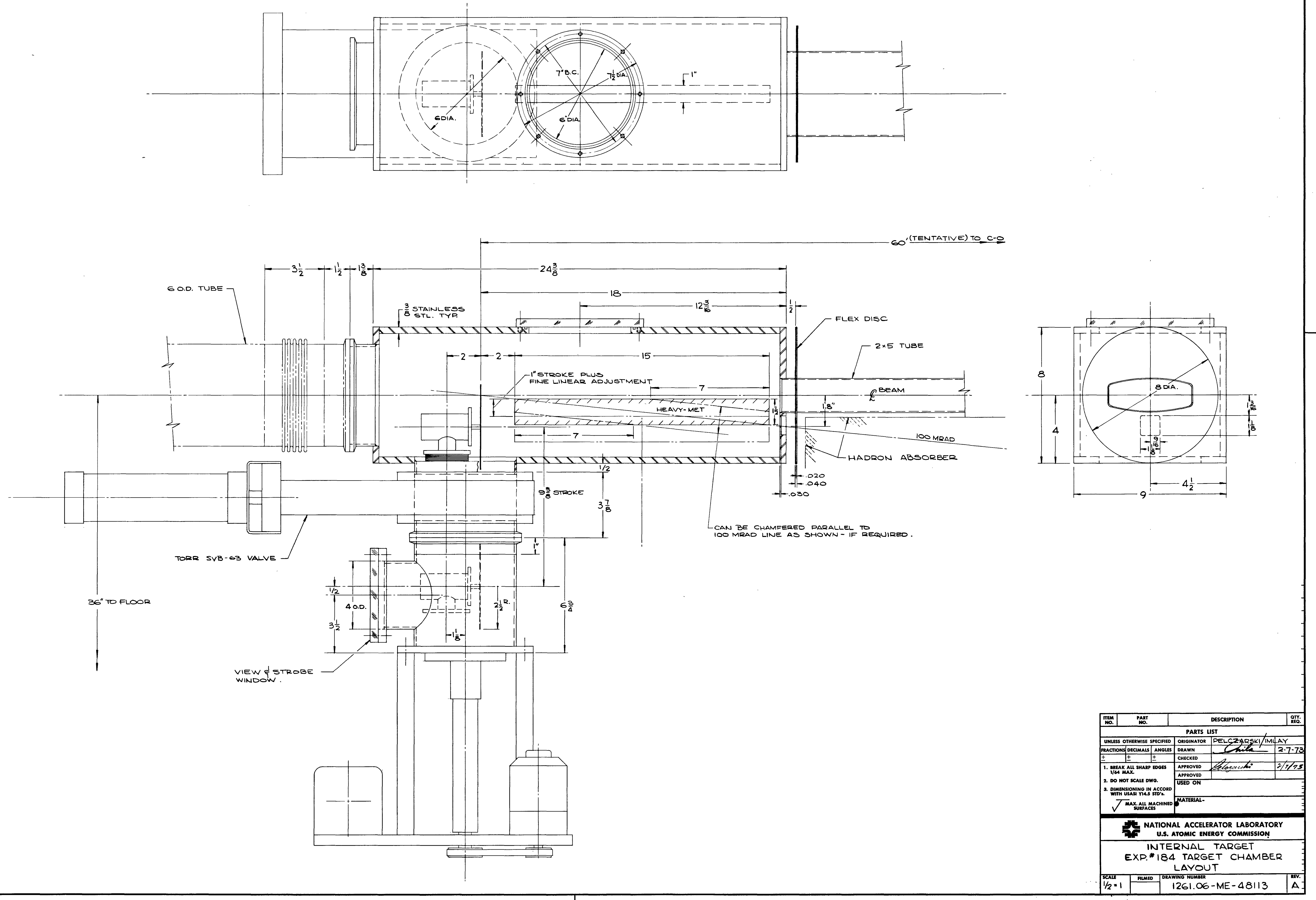